\title{
Electrical Properties Associated with Discharge Developments in Water Subjected to Impulse Voltages
}

\author{
Jong-Hyuk Choi ${ }^{\dagger}$ and Bok-Hee Lee*
}

\begin{abstract}
This paper describes electrical and optical characteristics of discharge developments in water under inhomogeneous fields caused by impulse voltages. Predischarge current and discharge light images were observed for different water resistivities and applied voltages between the hemispherical water tank and the needle electrode. The electrical parameters characterizing discharge developments are analyzed based on the discharge light images and voltage-current $(V-I)$ curves, and electrical resistances derived by voltage and current waveforms. As a result, when the streamer corona is initiated at the tip of the needle electrode, the transient resistance suddenly drops and $V-I$ curves form a 'loop'. The length of streamer propagation is increased with increasing peak value of the applied voltage, and the streamer corona extension is enlarged with increasing water resistivity. The electrical resistances before streamer corona initiation are rarely changed by different applied voltages. On the other hand, the electrical resistances after streamer corona initiation are found to be inversely proportional to the peak value of the applied voltage, and the decreasing rates for higher water resistivities are much higher than those for lower water resistivities. The time to streamer corona initiation and the time to the second current peak become shorter as the voltage increases. Finally, the calculated resistances after streamer corona initiation are almost the same trace of measured resistances, but they are smaller than the measured values.
\end{abstract}

Keywords: Streamer corona, underwater discharges, Predischarges, Breakdown phenomena, Discharge light images, Transient resistance

\section{Introduction}

There is considerable interest in the breakdown phenomena of water, particularly as a functional media. Streamer corona discharges in liquids can produce high energy electrons, ozone, chemically active species, ultraviolet rays and shock waves. The characteristics of electrical discharges in water are widely used in a variety of industrial, medical and environmental applications, as well as energy storage, sewage treatment, switching and insulation in pulsed power systems, water purification, etc. [1]-[3]. Gas and solid discharge phenomena have been investigated in detail and their physical properties are relatively well known. Also, the breakdown phenomena in insulating liquids have been studied for many years, but investigations into the nature of discharges in water have not been so numerous. Also, the characteristics of the breakdown caused by pulsed voltages in tap and distilled water with higher resistivities have been partially investigated [4], [5]. Although the mechanisms of streamer corona initiation and propagation in liquids are clearly not yet understood, two leading theories of the initial breakdown mechanisms in water are as follows: 1) charge injection occurs at the electrode/water

$\dagger \quad$ Corresponding Author: School of Electrical Engineering, Inha University, \#253, Yonghyun-dong, Nam-ku, Incheon, 402-751, Korea. (e-mail: chjohy80@naver.com).

* School of Electrical Engineering, Inha University, Incheon, Korea (e-mail:bhlee@inha.ac.kr).

Received : June 30, 2009; Accepted : January 13, 2010 interface, and 2) electron impact ionization occurs in a low-density region of the water [6]. Electrical discharges in water are useful not only to understand the physical properties of ionization phenomena in liquids but also to analyze the underwater discharges which occur in grounding electrodes immersed in ground water and rain water.

To clearly understand the electrical and optical properties of the discharge developments in water subjected to impulse voltages, more quantitative and detailed experimental results have to be considered. The objective of this paper is to present experimental data on the discharge phenomena and the parameters of the electrical breakdown in water subjected to impulse voltages. This paper presents electrical parameters in relation to the discharge developments in water under an inhomogeneous field caused by $1.2 / 50 \mu \mathrm{s}$ lightning impulse voltage in different water resistivities. The temporal characteristics of discharge developments are analyzed based on the discharge light images observed with a digital camera and a high speed ICCD camera simultaneously. Measured results and physical properties will be discussed in detail.

\section{Experiments}

To investigate the characteristics of electrical discharge development in water, the experimental set-up used in this work consisted of; the electrode system, the impulse voltage generator, and the measuring system, as shown in Fig. 
1. The voltage applied to the needle electrode was provided by the 8 -stage Marx generator producing a 1.2/50 $\mu \mathrm{s} \mathrm{im-}$ pulse waveform with a peak voltage of $400 \mathrm{kV}$. The maximum charge energy is approximately $20 \mathrm{~kJ}$ at $50 \mathrm{kV}$ charging voltage. The hemispherical water tank with a diameter of 1,100 mm was made of stainless steel. To take discharge light images, two observation windows with a diameter of $210 \mathrm{~mm}$ are installed at an angle of $90^{\circ}$ and a height of 150 $\mathrm{mm}$ from the bottom of the hemispherical water tank. The needle electrode was made of a stainless steel $3 \mathrm{~mm}$ diameter needle with a conical end. The radius of the tip of the needle electrode was $0.3 \mathrm{~mm}$. The needle electrode was elected at the center of the hemispherical water tank. Insulating and PVC pipes were used to support the needle electrode and enhance the insulating level. The needle electrode was immersed in the test vessel filled with water to a depth of $200 \mathrm{~mm}$. In this experiment, the separation between the tip of the needle electrode and the bottom of the hemisphere water tank was fixed at $100 \mathrm{~mm}$. All experiments were carried out at room temperature.

The voltage at the needle electrode, and the current through the test gap, were measured by the capacitive divider with 10,000:1 ratio and a $0.02 \Omega$ sensitive shunt, respectively, and their waveforms are recorded at a digital storage oscilloscope which has 4 channels and $400 \mathrm{MHz}$ bandwidth. Discharge light images were taken by a digital camera and fast gated ICCD camera which have minimum $50 \mathrm{~ns}$ exposure time. To take discharge lighting images clearly, the hemispherical water tank is compassed by dark cloth. To evaluate the electrical discharge characteristics in tap water and rain water, water resistivity was adjusted in the range from $10 \Omega$. m to $200 \Omega$. m by adding sodium chloride $(\mathrm{NaCl})$ and measured by the electric conductivity meter (CM-21P, TOA). The resistances before and after streamer corona initiation at the tip of the needle electrode were analyzed in different water resistivities as a function of applied voltages. The calculated resistances after streamer corona initiation, which are obtained using the length of streamer corona propagation measured by the time-integrated discharge light images, were compared with measured values.

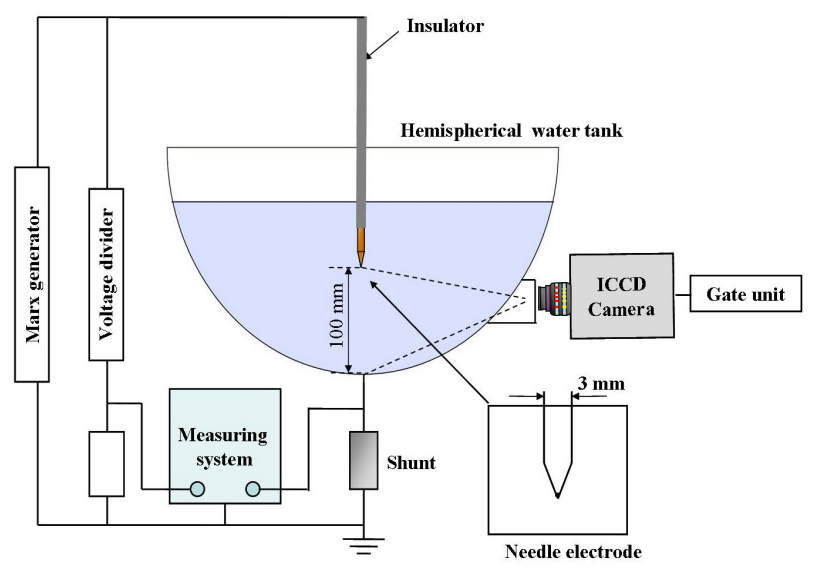

Fig. 1. A schematic diagram of the experimental set-up.

\section{Results and Discussion}

\subsection{Characteristics of streamer discharges}

Streamer corona discharges in water occur when a very high electric field is suddenly applied to the water gap. An electron avalanche can occur immediately in water under a high electric field, leading to the propagation of the water streamer [7]. Streamer corona initiation and propagation mechanisms in some liquids have been reported, two models for streamer corona initiation are considered: one is the electronic process, which involves electron injection drift in the liquid at the cathode, the other covering the concept of microbubble formation due to vaporization and/or cavitation and electrical breakdown in the gas bubbles [8], [9]. Fig. 2 shows the time-integrated discharge light images in water, taken by the digital camera, for different voltages and water resistivities. Because the discharge light images caused by streamer corona are faint, the digital camera was set to an exposure time of 5 seconds and 5.6 irises during the experiment. When the applied voltage in the positive polarity is gradually increased from zero, streamer coronas begin to appear at the tip of the needle electrode and propagate toward the bottom of the hemispherical water tank.

At the water resistivity of $200 \Omega . \mathrm{m}$, the streamer corona initiates from $31.8 \mathrm{kV}$ to $50.4 \mathrm{kV}$ and the electrical breakdown occurred at voltages greater than $50.4 \mathrm{kV}$. The streamer corona length increases with an increase in the magnitude of the applied voltage. The streamer coronas which occurred at the level below the electrical breakdown voltage cease in water. When water resistivity decreases, the streamer corona initiation voltage is lowered, but the electrical breakdown voltage is elevated. The streamer corona developments in water are principally electrical and optical processes which are caused by the electron avalanche effects of the air in the bubbles. It has been reported previously that the streamer corona development mechanisms in water are closely associated with the ionization of the air in the bubbles [10]. There is immediately a striking resemblance between propagating electrical trees in solids and streamer coronas in liquids. Light emission may be expected to occur, being produced both in the bond breaking processes and also from molecular excitations accompanying the electron acceleration in the electric field. The cathode-directed streamer corona propagation from the anode arises from the creation of a new liquid/air interface and the reilluminations observed in the streamer do not have conductive properties, and are not necessarily connected back to the anode [9]. Also, the difference between the critical streamer corona initiation voltage and the electrical breakdown voltage increases with decreased water resistivity. Photographs at the right end of Fig. 2 show examples of the voltages just below the electrical breakdown voltage in water for various resistivities. The timeintegrated discharge light images, taken by a digital camera, for lower water resistivities illustrate the cathode-directed streamer corona propagation, and are much thicker and 

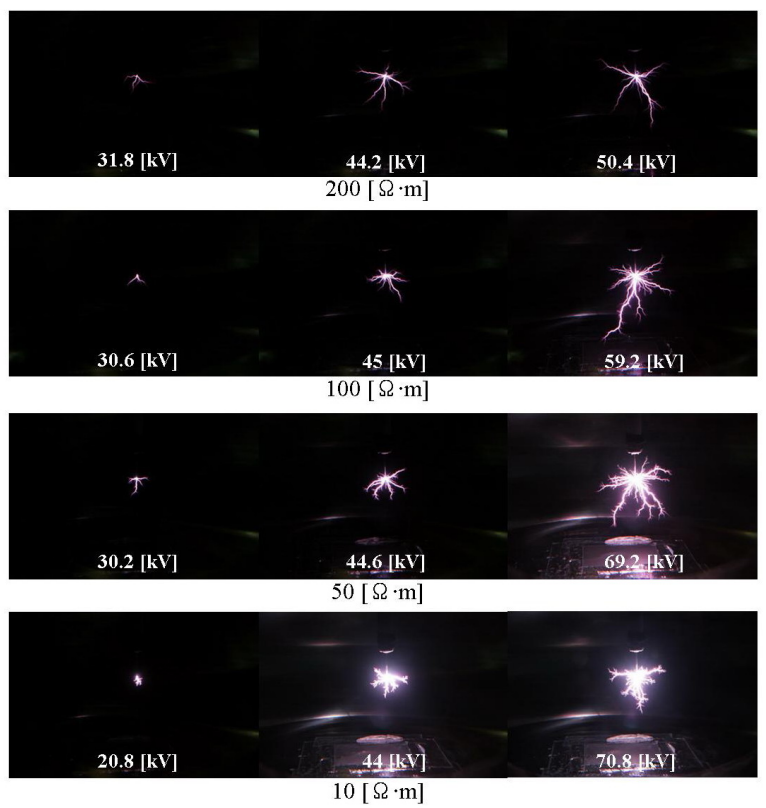

Fig. 2. Time-integrated discharge light images caused by streamer corona discharges for different water resistivities and applied voltages.

brighter than those for higher water resistivities at the same level of applied voltage.

The dependence of streamer corona development on the applied voltages was measured. A sketch of the crosssectional view of the ionized region around the tip of the needle electrode was shown in Fig. 3. The length of streamer corona discharge around the tip of the needle electrode in radial directions was not uniform. The streamer corona expands intensively in the axial direction of the needle electrode in comparison with radial size because of the conical end of the tip of the needle electrode. The length of the visual streamer corona increases with increasing applied voltage, until the electrical breakdown occurs, as reported in the literature [11].

The streamer length was obtained from the timeintegrated discharge light images taken by the digital camera. Fig. 4 shows the variation of the maximum streamer length for different water resistivities as a function of peak voltage. Although the data are dispersed, the length of the

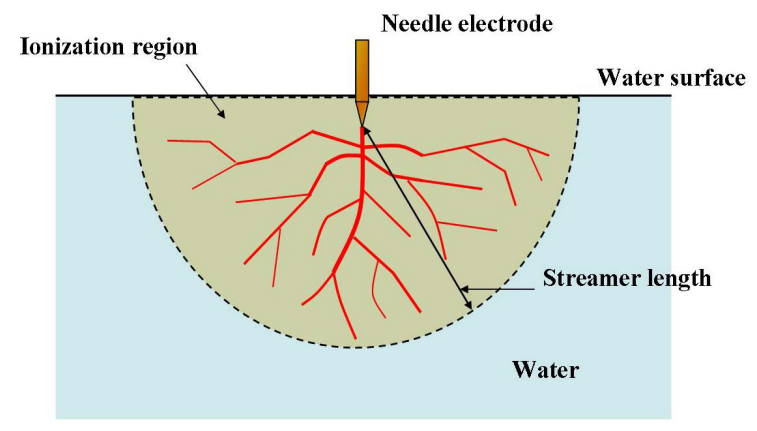

Fig. 3. A sketch of the cross-sectional view of the ionization region around the tip of the needle electrode.

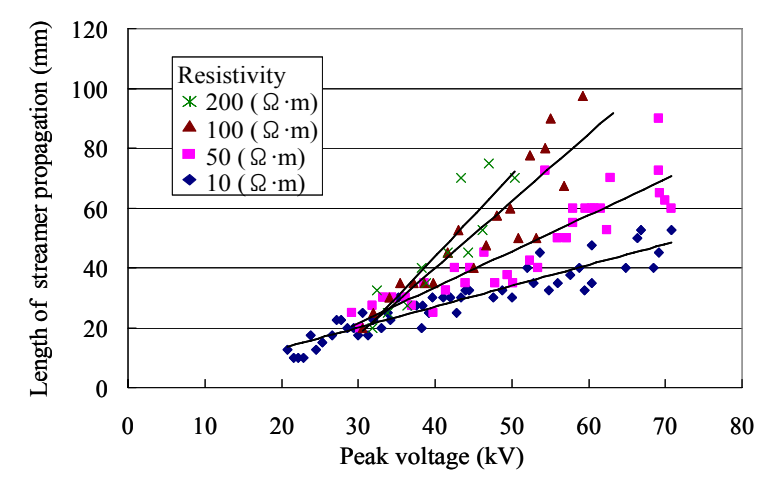

Fig. 4. The length of streamer corona propagation as a function of peak voltage.

streamer corona development is roughly proportional to the peak voltage. The dependence of the length of streamer corona development on the peak voltage is dominant for higher water resistivity. During the streamer propagation, numerous micro-bubbles are produced not only in the vicinity of the leader stem but also in the tip of the streamer corona. The size of the bubble must be sufficient for the growth of the streamer corona and the conversion into a fast breakdown streamer according to the theory of discharge in liquids in the microsecond time [12].

\subsection{Electrical Parameters}

When the electrical characteristics of streamer corona discharge developments in water are evaluated, ionization phenomena need to be taken into account. Two methods were used, in this paper, to analyze these characteristics and phenomena. Typical examples of the measured voltage and current waveforms in water with resistivity of 50 $\Omega \cdot \mathrm{m}$ for different applied voltages were illustrated in Figs. 5 (a) and (b), and the transient resistance traces derived from dividing the voltage by current were represented in Fig. 5 (c). The current flow through the test gap can be seen to occur in two stages: (1) pre-ionization, in which the current depends on the water resistivity, and (2) post-ionization, characterized by a large current originated from the streamer corona initiation. The step or plateau on the leading edge of the current pulse was observed at voltage levels greater than the streamer corona initiation voltage. The plateau is produced, in this case, by the combination of different conduction mechanisms. Since the initial rising part of the leading edge of the current pulse is proportional to the magnitude of applied voltage, it is caused by an ionic conduction mechanism. The second rising part of the current pulse will be caused by the transition of an ionic conduction mode to a streamer corona discharge mode. That is, the nonlinearity is attributed to the streamer corona propagation processes due to the electric field enhancement in the vicinity of the tip of the needle electrode.

The electrical resistance at the applied voltage of $30 \mathrm{kV}$ is rarely changed, since the current increases linearly with voltage. On the other hand, at an applied voltage of $60 \mathrm{kV}$, 
the ionization is initiated at the time of $5 \mu \mathrm{s}$, then the current rises and the voltage drops steeply. The higher the applied voltage is, then the shorter the time to the ionization threshold. The temporal resistance, defined as the voltage divided by the current, also gets lower with increasing magnitude of applied voltage. The temporal resistance at the instant of the ionization threshold drops sharply, as shown in Fig. 5 (c). Because the ionization region, shown in Fig. 3, is likely to act as an expended electrode, the electrical resistance is significantly decreased to a value less than the initial static resistance. For example, the electrical resistance at the applied voltage of $80 \mathrm{kV}$ decreases from $800 \Omega$ to $200 \Omega$ for a time of $3 \mu \mathrm{s}$.

Ionization characteristics in water were also analyzed by $V-I$ curves for different applied voltages and water resistivities, as plotted in Figs. 6 (a) and (b), respectively. The $V-I$

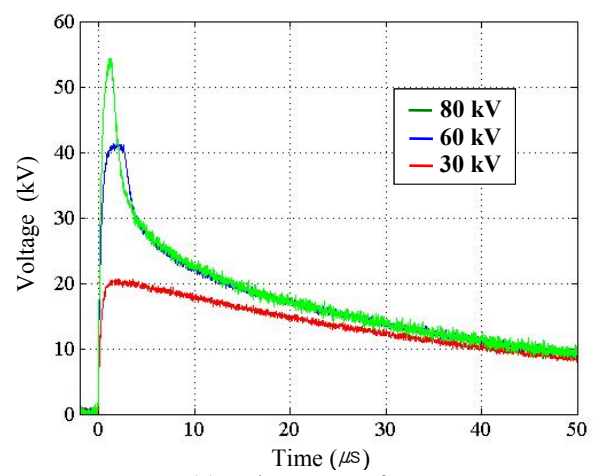

(a) Voltage waveform

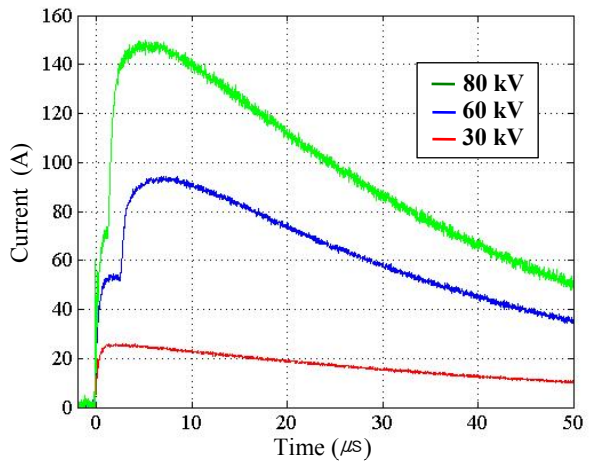

(b) Current waveform

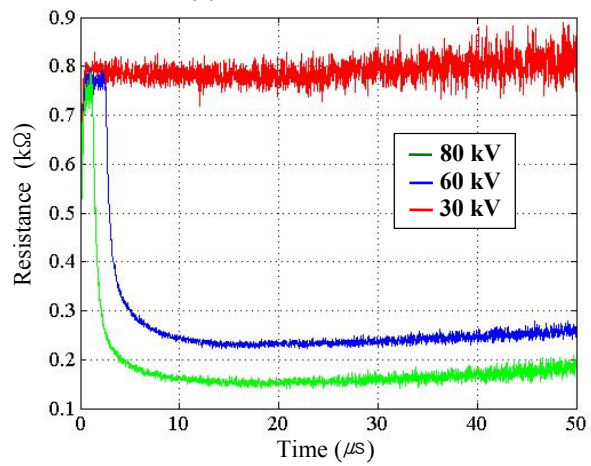

(c) Temporal variation of resistance

Fig. 5. Waveforms of the voltage, current and transient resistance in water with the resistivity of $50 \Omega \cdot \mathrm{m}$ for different applied voltage. curves are strongly dependent on the water resistivity and the magnitude of applied voltages.

At voltages less than the ionization threshold, the current linearly increases with the magnitude of applied voltage, and the $V-I$ curve is a straight line, as seen in Fig. 6 (a). On the other hand, as the magnitude of applied voltage is higher than the ionization threshold level, the $V-I$ curves form a 'loop' due to the difference in front and tail times between the voltage and current traces. The $V-I$ curves are drawn clockwise and their sizes get larger with increasing magnitude of applied voltage. The slopes of the straight line drawn between the origin and each point on the $V-I$ curves mean the electrical resistance and the clockwise drawn loop represents a decrease of electrical resistance.

Fig. 6 (b) illustrates the variation of the $V-I$ curve as a parameter of the water resistivities at the applied voltage of $80 \mathrm{kV}$. All $V-I$ curves form loops. The maximum voltage and current for the water resistivity of $100 \Omega \cdot \mathrm{m}$ were 62 $\mathrm{kV}$ and $125 \mathrm{~A}$, respectively. However, in the case of the water resistivity of $10 \Omega \cdot \mathrm{m}$, the maximum voltage decreases to $28 \mathrm{kV}$ and the maximum current increases to 155 A. As the water resistivity is decreased, the loops are rotating clockwise, which mean that the electrical resistance after ionization decreases. The $V-I$ curve for the water resistivity of $10 \Omega \cdot \mathrm{m}$ illustrates the two step development of streamer corona discharges. Because the ionic conduction currents for lower water resistivities are relatively large compared to the current resulting from streamer corona discharge, and the size of the loop is getting smaller.

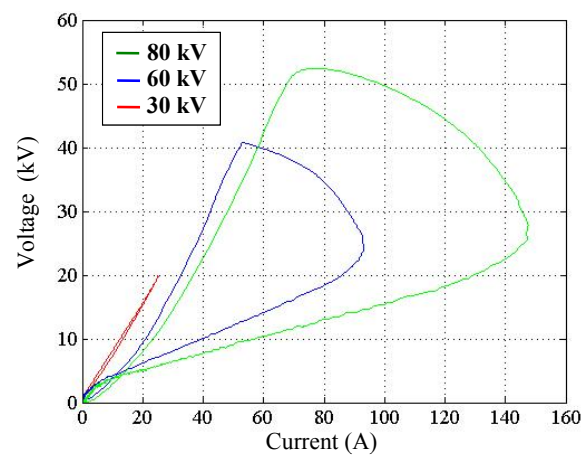

(a) For different applied voltages

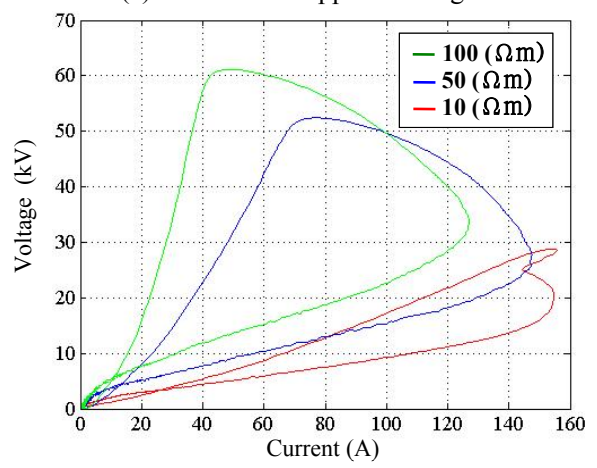

(b) For different water resistivities

Fig. 6. $V-I$ curves as parameters of water resistivity and applied voltage. 
When recording the voltage and current waveforms, discharge light images were taken by a digital camera and a high speed ICCD camera simultaneously. Fig. 7 shows typical examples of the measured voltage and current waveforms and the time-integrated and temporal discharge images observed with the digital camera and ICCD camera with a time interval of 500ns at the initial $2 \mu \mathrm{s}$ delay time. The discharge light images illustrate the cathode-directed streamer corona propagation. The discharge light images will tend to be synchronous with the current pulses. The sudden voltage drop and current rise due to exceeding the ionization threshold coincide with the appearance of the discharge light images which appear after $4 \mu \mathrm{s}$ in Fig. 7.

The current waveform at a voltage just above the streamer corona initiation voltage is given with a step on the leading of the pulse in the predischarge regime. The current pulses have two current peaks with some time-lags. To analyze the ionization phenomena, the two time parameters are defined as shown in Fig. 7 (b): where the time-lag $t_{1}$ is the time to ionization threshold (preionization time) and the time-lag $t_{2}$ is the time to the second current peak (post-ionization time). Also, the preionization resistance $R_{1}$ is defined as the voltage divided

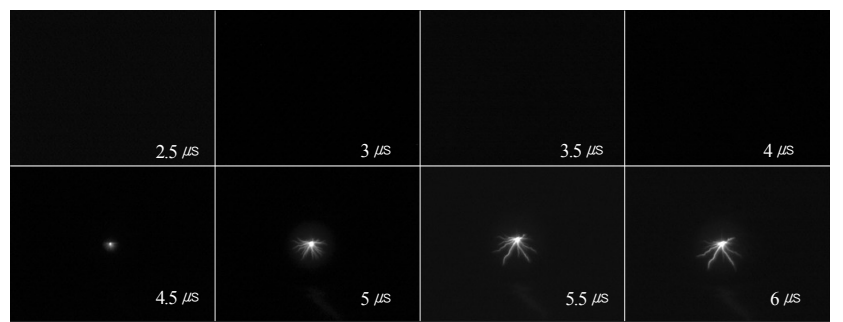

(a) Discharge light images for temporal developments of streamer corona discharges in water observed with an ICCD camera

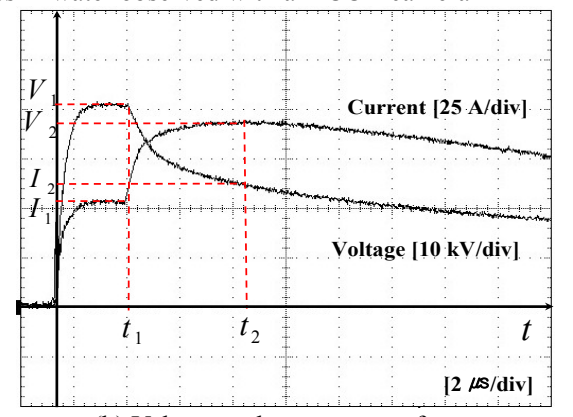

(b) Voltage and current waveforms

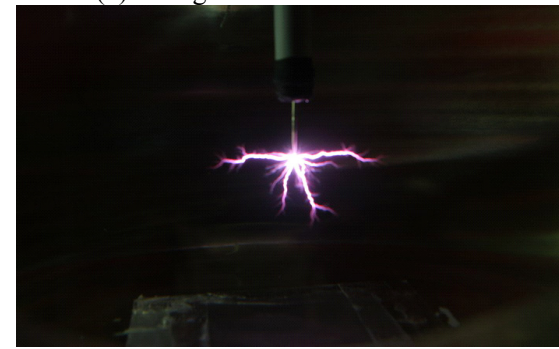

(c) Time-integrated discharge light image observed with digital camera

Fig. 7. Discharge light images, voltage and current waveforms recorded simultaneously. by the current at the time of $t_{1}$ and the post-ionization resistance $R_{2}$ is calculated by the same method at time $t_{2}$.

Fig. 8 shows the results of the time-lag to the ionization threshold $t_{1}$ and the time-lag to the second current peak $t_{2}$ as a function of peak voltage. The time-lag $t_{1}$ for the streamer corona initiation after voltage application was sensitive to the magnitude of applied voltage, but it was insensitive to water resistivity. Both time-lags $t_{1}$ and $t_{2}$ were inversely proportional to the applied voltage. The time-lags $t_{1}$ tends to be shorter with increasing water resistivity, but the time-lags $t_{2}$ for higher water resistivities were longer than those for lower water resistivities at the same magnitude of applied voltage. The diverted result of the time parameter came from the variations of the conduction currents and the difference between the applied voltage and the peak voltage.

Ionization in water is caused by electric field enhancement at the tip of the needle electrode. The ionization leads to the creation of some parallel streamer coronas propagating away from the energized electrode. The length of the streamer corona propagation is closely dependent on the applied voltage. The average streamer corona propagation velocity is constant during the voltage application and has almost identical values for different voltages [8]. When streamer coronas propagate radically toward the grounded water tank, the electrical resistance of the ionization region will fall from high to low values.

The post-ionization resistance could be calculated using the assumptions that replace the needle electrode with expended hemispherical electrode and substitute ionization

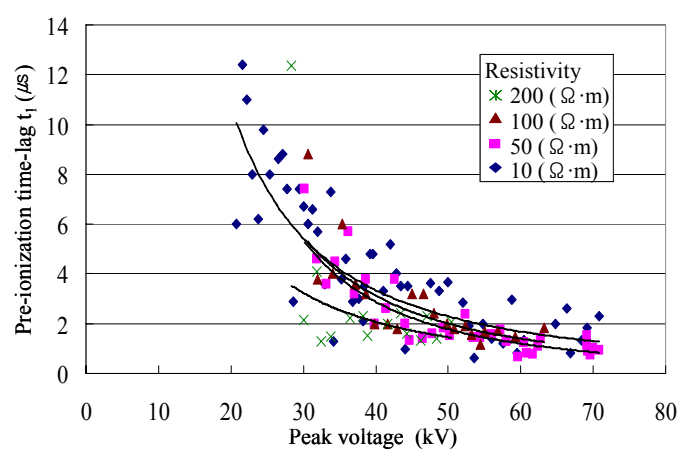

(a) Pre-ionization time-lag

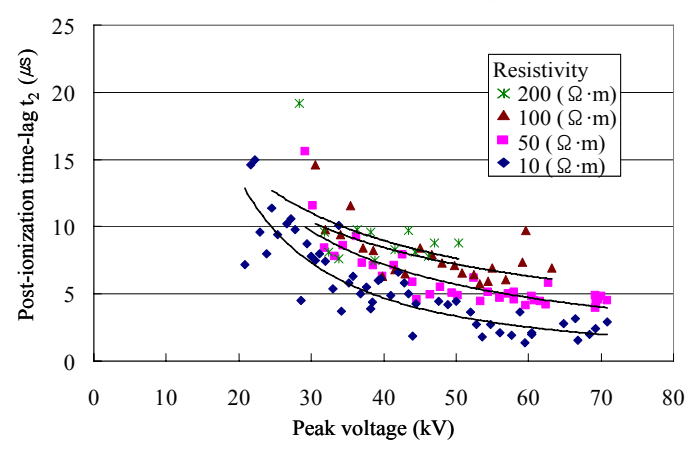

(b) Post-ionization time-lag

Fig. 8. Time parameters for different peak voltages. 
radius by the maximum length of streamer propagation as shown in Fig. 4. Assuming that the ionization region expands uniformly, the post-ionization resistance could be calculated from Eq. (1).

$$
R_{2}^{\prime}=\frac{\rho}{2 \pi}\left(\frac{1}{r_{1}}-\frac{1}{r_{2}}\right)
$$

where,

$r_{1}$ is radius of the ionization region

$r_{2}$ is radius of the hemispherical water tank

The pre-ionization resistance $R_{1}$, the measured postionization resistance $R_{2}$ and the calculated post-ionization resistance $R_{2}{ }^{\prime}$ for different peak voltages and water resistivities were shown in Fig. 9. Because the voltages increase linearly with current, the pre-ionization resistances $R_{1}$ are not seriously affected by peak voltages. The pre-ionization resistance $R_{1}$ for the water resistivity of $200 \Omega \cdot \mathrm{m}$ was about $3 \mathrm{k} \Omega$ and it was proportionally reduced to less than $180 \Omega$ for a water resistivity of $10 \Omega \cdot \mathrm{m}$. On the other hand, the measured post-ionization resistances $R_{2}$ for different applied voltage are lower than half of the preionization resistances at the same applied voltages. As expected, the post ionization resistances $R_{2}$ are inversely proportional to peak voltages because the length of the streamer propagation increases with increasing the applied voltages. When ionization is initiated, the ionization region around the tip of the needle electrode, operating as a radically expended electrode, decreases the post-ionization resistance. The reducing rate of the post-ionization resistance for higher water resistivities were larger than that for lower water resistivities, as the conduction currents increase sharply with decreasing water resistivity. The calculated post-ionization resistance $R_{2}{ }^{\prime}$ shows the same tendency with the variation of $R_{2}$, however the resistance $R_{2}{ }^{\prime}$ is lower than the resistance $R_{2}$.

The difference between the resistances $R_{2}{ }^{\prime}$ and $R_{2}$ comes from the error factors in the expended ionization region caused by streamer discharges not being correct hemispherical shapes and the maximum length of streamer propagation was employed as the radius of expended ionization region. It is considered that the difference between the measured and calculated results could be originated from the intensive streamer expansion in the axial direction of the needle electrode with a conical end. In this analysis, it has been assumed that the ionization in water takes place uniformly around the tip of a needle electrode. However, practically, the electric field is enhanced at the tip of a needle electrode, and it is probable that the ionization is initiated at the tip of a needle electrode and is strongly developed near the needle electrode.

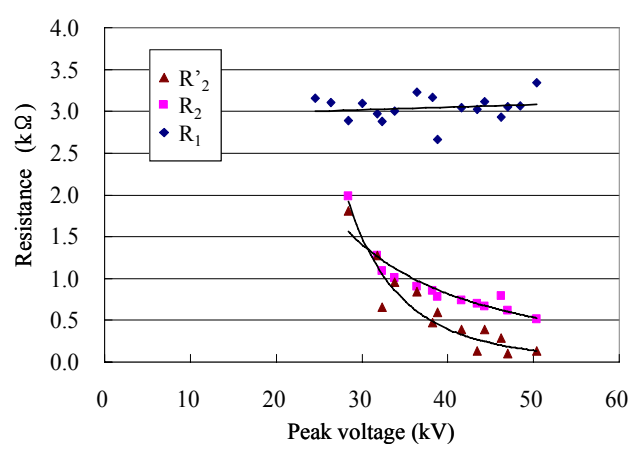

(a) $200[\Omega \cdot m]$

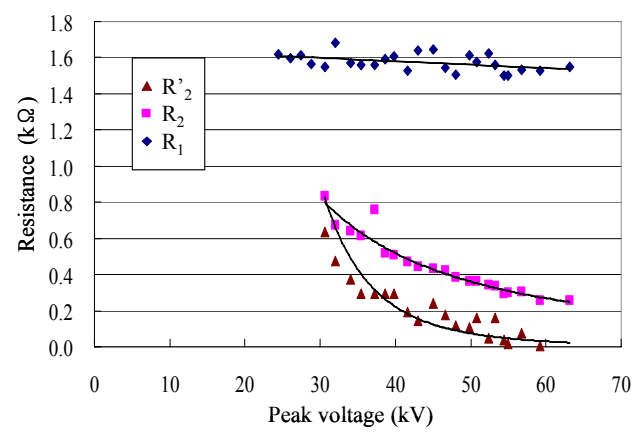

(b) $100[\Omega \cdot \mathrm{m}]$

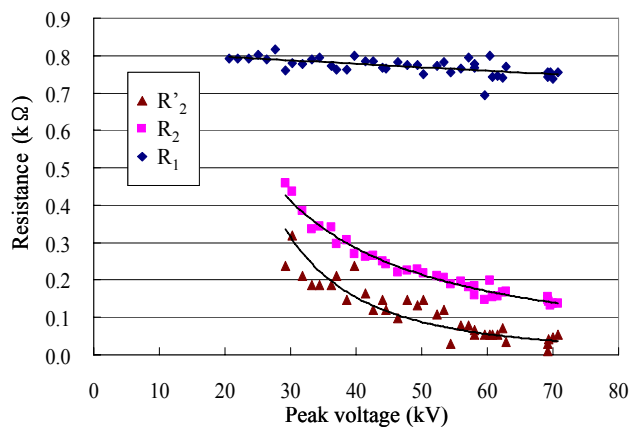

(c) $50[\Omega \cdot \mathrm{m}]$

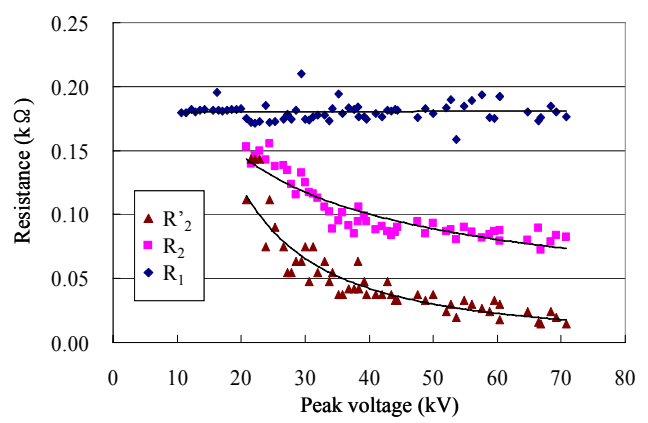

(d) $10[\Omega \cdot \mathrm{m}]$

Fig. 9. Resistance $R_{1}, R_{2}$ and $R_{2}{ }^{\prime}$ as a function of the peak voltage for different water resistivities.

\section{Conclusions}

This paper has investigated the electrical and optical characteristics of the streamer corona discharge developments in water initiated at the tip of a needle electrode subjected to standard lightning impulse voltage. The streamer 
corona discharge developments in water have been analyzed by not only the discharge light images but also voltage/current waveforms, transient electrical resistance variations and $V-I$ curves. The experimental results obtained in this work could be summarized as follows:

As the water resistivity decreases, the ionization threshold voltages are gradually reduced and the discharge light images are more dispersed and brighter. The two separate conduction phases were observed above a certain voltage level, and the second current peak is produced by the streamer corona initiation at the tip of the needle electrode. It was found that the time-lag to the ionization threshold and the time-lag to the second current peak were shortened with the magnitude of applied voltages. The $V-I$ curves above the ionization threshold voltage form a 'loop', the size of the loop is reduced with decreasing water resistivity. The post-ionization resistance above the ionization threshold voltage was significantly reduced due to expanding ionization regions. The calculated post-ionization resistances have good correlation with the measured results in all water resistivities. It is believed that the asymmetric streamer expansion is the main reason for the difference between the measured and calculated resistances.

\section{Acknowledgements}

This work was supported by the Ministry of Knowledge Economy under Grant R-2007-1-014.

\section{References}

[1] T. Namihira, S. Sakai, T. Yanaguchi, K. Yanampyo, C. Yamada, T. Kiyan, T. Sagugawa, S. Katsuca, and H. Akiyama, "Electron Temperature and Electron Density of Underwater Pulsed Discharge Plasma Produced by Solid-State Pulsed-Power Generator," IEEE Trans. On Plasma Science, Vol.35, No.3, pp.614-618, Jun., 2007.

[2] H. Akiyam, "Streamer discharge in liquids and their applications," IEEE Trans. DEI, Vol.7, No.5, pp.646653, 2000.

[3] X. Lu, Y. Pan, K. Liu, M. Liu, and H. Zhang, "Spark model of pulsed discharge in water," J. Appl. Phys., Vol.91, No.1, pp.24-31, 2002.

[4] H. M. Jones and E. E. Kunhardt, "The Influence of Pressure and Conductivity on the Pulsed Breakdown of Water," IEEE Trans. DEI, Vol.1, No.6, pp.10161025, 1994.

[5] Y. H. Sun, Y. X. Zhou, M. J. Jin, Q. Liu, and P. Yan, "New prototype of underwater sound source based on the pulsed corona discharge," J. Electrostatic, Vol.63, pp.969-975, 2005.

[6] D. A. Wetz, K. P. Truman, J. J. Mankowski, and M. Kristiansen, "The Impact of Surface Conditioning and Area on the Pulsed Breakdown Strength of Water," IEEE Trans., on Plasma Science, Vol.33, No.4, pp.1161-1169, Aug., 2005.

[7] G. Touya, T. Reess, L. Peecastaing, A. Gibert, and P. Domens, "Development of subsonic electrical discharges in water and measurements of the associated pressure waves," J. Phys. D., Appl. Phys. Vol.39, pp.5236-5244, 2006.

[8] S. Katsuki, H. Akiyama, A. Abou-Ghazala, and K. H. Schoenbach, "Pararrel Streamer Discharges Between Wire and Plane Electrodes in Water," IEEE Trans. On Dielectrics and Electrical Insulation, Vol.9, No.4, pp.498-506, 2002.

[9] T. J. Lewis, "A New Model for the Primary Process of Electrical Breakdown in Liquids," IEEE Trans. On Dielectric and Electrical Insulation, Vol.5, No.3, pp.306-315, Jun., 1998.

[10] J. Nieto-Salazar, O. Lesaint, and A. Denat, "Transient current and light emission associated to the propagation of pre-breakdown phenomena in water," Proc. 2003 Annual Report Conference on Electrical Insulation and Dielectric Phenomena, pp.542-545, 2003.

[11] P. Bruggeman, C. Leys, and J. Vierendeels, "Experimental investigation of dc electrical breakdown of long vapour bubbles in capillaries," J. Phys. D., Appl. Phys., Vol.40, pp.1937-1943, 2007.

[12] A. T. Sugiarto, M. Sato, and J. D. Skalay, "Transient regime of pulsed breakdown in low-conductive water solutions," J. Phys. D., Appl. Phys., Vol.34, pp.34003406, 2001.

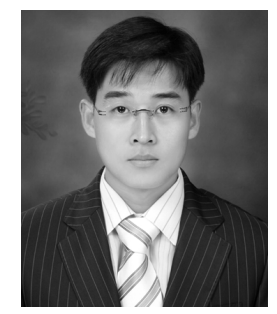

Jong-Hyuk Choi was born in Incheon, Korea, in 1980. He received his B.S. degrees in Electronic Engineering from Inha University in 2006. He received his M.S. degree in Electrical Engineering from Inha University, in 2008. He is currently working toward his Ph. D. degree in the School of Electrical Engineering, Inha University, Incheon, Korea. His research interests include high voltage engineering, grounding, underwater discharge, lightning protection.

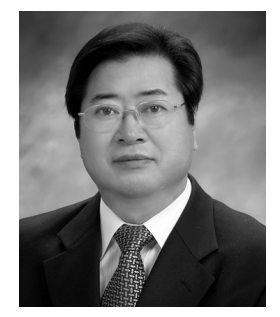

Bok-Hee Lee received his Ph.D. degree in Electrical Engineering from Inha University in 1987 . He has been with the school of Electrical Engineering at Inha University, Inchon, Korea, as an Assistant Professor since 1990, becoming a Professor in 1999. From 1988 to 1989 , he was a post-doctoral research fellow at the Institute of Industrial Science, University of Tokyo. From Apr. 1999 to Feb. 2000, he was a Visiting Professor in the University of Cincinnati. His research interests are in the area of lightning, lightning protection, grounding systems, surge protection, electrical discharges, high voltage engineering and electromagnetic compatibility. 